\title{
DIAGNÓSTICO GEOECOLÓGICO COMO BASE PARA EL ORDENAMIENTO DE UN TERRITORIO: PARQUE METROPOLITANO DE LA HABANA
}

\author{
por \\ DIAZ GARCIA, S. \\ (Facultad de Geografia. Universidad de La Habana, Cuba.)
}

\section{RESUMEN}

El crecimiento de las ciudades reduce el potencial de espacios verdes en ellas, por lo que la demanda de espacios de uso recreativo, con tendencia a resaltar los valores naturales y estéticos, deben ser considerados en la planificación y gestión de aquellos lugares que aún conserven un valor ambiental.

Teniendo en cuenta esas premisas, se crea el Parque Metropolitano de La Haba$n a$, en el que se elabora una zonificación basada en un mapa de unidades de paisaje que son analizadas mediante indicadores que permiten establecer el diagnóstico geoecológico de todo el territorio, y programar medidas para su ordenamiento.

Palabras Glave: espacios verdes, valores estéticos y ambientales, zonificación, paisajes, diagnóstico y geoecológico.

\section{ABSTRACT}

The development of the cities decrease the potencial of green areas. The population require that spaces for its amusement and recreation and also in order to be evident the esthetics and natural values. These aspects will be considered in the planning and management of those places that still keep environment value.

According with those ideas the public administration of the city put up the Metropolitan Park of Habana. Its territory had been divided into units of landscape and studied by some gauges or index which allowed to establish the geoecological diagnostic of the territory and to program measures for its planning.

KeY WORDS: green areas, esthetics and environment values, landscape, geoecological diagnostic. 


\section{INTRODUCCIÓN}

Ante el auge del crecimiento de la ciudad de La Habana en su expansión hacia el oeste, se concibe en las primeras décadas del siglo $\mathrm{XX}$, la delimitación de un amplio espacio para uso público en los terrenos próximos al río Almendares. El grado de conservación de los recursos naturales de dicho espacio, así como su alto valor ambiental, propiciaban la creación de un gran parque que sirviera de recreación y entretenimiento a los habitantes de la ciudad, a la vez que se conservaban sus valores naturales y estéticos.

Por diversas razones, el proyecto fue postergado en varias ocasiones y no es hasta casi finales de siglo cuando se comprende la necesidad de revitalizar dicho proyecto. Así, la Administración de la ciudad aprueba, en 1.996, la creación del Parque Metropolitano de La Habana; el espacio destinado al parque ya había adquirido características singulares.

La presión de uso sobre el suelo y sus recursos redujo la extensión del área inicialmente proyectada en una proporción notable, ya que muchas porciones de suelo fueron destinadas a actividades muy diferenciadas como agricultura, pastoreo de ganado menor, industrias contaminantes de variado tipo, sectores residenciales, población marginal, almacenes y talleres, y también, lugares de recreación y esparcimiento. Las con- secuencias del uso descontrolado del territorio tuvo varias manifestaciones, destacándose en primer lugar el elevado grado de contaminación del río Almendares, a lo que se añadió la deforestación, erosión de los suelos, contaminación ambiental, y otras acciones negativas.

Al retomar la idea del parque, se hizo evidente la necesidad de un reordenamiento del territorio, previo conocimiento del impacto provocado por las acciones antrópicas. Para ello era preciso realizar un diagnóstico geoecológico que permitiera establecer los grados de degradación a los que se había llegado. De esa manera, se podría estudiar de forma multidisciplinaria, conjuntamente con todos los intereses que conforman el territorio, un sistema de medidas para su manejo y gestión.

\section{ESTRUCTURA CONCEPTUAL DE UN PARQUE METROPOLITANO}

El eje principal del Parque Metropolitano de La Habana es el río Almendares, cuyo territorio muestra una multifuncionalidad que crea conflictos de uso.

El reordenamiento del parque requiere la elaboración de una zonificación de usos del suelo por áreas. La definición de esas áreas es de vital importancia, puesto que deben ser valoradas conforme a su estructura, funcionamiento $y$ dinámica, de acuerdo con la función o 
funciones que dichas áreas deban desempeñar. Con base en las valoraciones realizadas es posible elaborar propuestas para establecer un sistema de medidas que englobe el conjunto de acciones encaminadas a garantizar el mejoramiento de las características de dichas áreas, para cumplir las funciones que les han sido asignadas en el modelo de planificación, ya sean de aprovechamiento, protección, conservación o rehabilitación.

La valoración de las áreas se hizo según los principios de la Geoecología de los Paisajes, por las ventajas que ofrece para el análisis conceptual y metodológico. La Geoecología es una ciencia moderna que se nutre de concepciones sistémicas a partir de la Geografía y la Ecología. Es decir, reconceptualiza las nociones de geosistema y ecosistema y las interpreta como diferentes niveles de organización del espacio. No hay contradicción entre ellos: el ecosistema comprende una parte del geosistema al cual se haya vinculado, de manera que aquél recibe de éste una dimensión espacial, mucho más amplia. Mientras en el ecosistema se percibe como un centro (objeto de estudio), en relación con el entorno más cercano para determinar su funcionamiento, el geosistema le proporciona la dimensión espacial estableciendo sus límites.

Los geosistemas se concretan por su estructura, funcionamiento y dinámica, y su tipología viene dada por el factor que se escoge como objeto. De tal suerte que la función del geoecosistema es asumir un centro biótico, es decir, por el ecótopo, incluido en un fondo sistémico.

La Geoecología presta máxima atención a la circulación de materia y energía entre los componentes de una unidad geográfica dada, la cual es operacional y se denomina paisaje o complejo territorial natural, que constituye en sí misma un complejo integrador de los componentes del medio geográfico que interactúan entre sí y tienen capacidad para formar una estructura espacial de diferentes niveles de sistematización (Mateo, 1.984).

De esta manera, en el paisaje, como sistema conceptual, se produce una relación objeto-sujeto que, pasando por filtros de percepción, determinan:

-Su objetividad.

-Su transdisciplinariedad.

-El tipo y estructura del sistema.

-El análisis multidisciplinar.

-Su realidad tangible.

Así, la investigación geoecológica descansa en la interacción entre la naturaleza y la sociedad. Ello permite conocer la regularidad en la génesis, desarrollo y diferenciación espacial y temporal de los paisajes; lo cual incluye el análisis histórico natural de los mismos, vistos como formaciones antroponaturales contemporáneas (ACEVEDO, 1.996). 
Visto así, el paisaje como formación antroponatural, consiste en un sistema territorial compuesto por elementos naturales y antropo- tecnógenos que, condicionados socialmente, modifican y transforman las propiedades de los paisajes naturales.

\section{ORDENAMIENTO GEOECOLÓGICO DEL PARQUE METROPOLITANO DE LA HABANA*}

Partiendo de una fase primaria organizativa, se pasó a una etapa de inventario en la que se precisó la selección de las unidades operacionales, determinádose así las unidades de paisaje. Para ello se elaboró un Mapa de Paisajes, a partir del mapa tipológico a escala 1:10.000, que representa una diversidad importante de individuos, en el cual se delimitaron 10 comarcas y 31 subcomarcas. Este mapa se basa en la relación e interacción de los componentes del medio geográfico en su estado natural.

Luego, mediante el análisis, se utilizaron varios indicadores que permitían caracterizar las diferentes unidades. Los indicadores utilizados fueron :

- La estabilidad del paisaje, que descansó en el estudio de las estructuras vertical, horizontal y funcional. La estabili- dad es inherente a la organización del paisaje.

- El siguiente indicador fue el uso del suelo, que permitió definir el uso que tiene asignada cada unidad de paisaje. Ello permitió utilizar el indicador de modificación antrópica mediante la utilización del Coeficiente de Modificación Antrópica.

- A continuación, se procedió a determinar la vulnerabilidad o sensibilidad de los paisajes. A menor estabilidad natural y mayor grado de modificación antrópica, los paisajes son más sensibles o vulnerables, pudiendo determinarse una gradación para este indicador.

La determinación de la vulnerabilidad dio paso al funcionamiento de las unidades de paisaje; es decir, se pudo precisar el patrón general de funcionamiento local, considerando las condiciones de los componentes - y el grado de antropización de las unidades de paisaje, lo que conforma un complejo funcional coherente.

Se siguió con los procesos geoecológicos determinándose los predominantes en cada área. El estado geoecológico fue utilizado analizando los

* Nota del Editor: En el trabajo original se inclúan cuatro anexos y tablas que hacían referencia a los diferentes indicadores utilizados, pero no se pudieron abrir al ser transmitidos por e-mail. 
indicadores anteriores, y se expresó mediante categorías que dependen de la pérdida de la capacidad de reproducción de los geosistemas al ser modificados sus atributos y propiedades esenciales.

La evaluación de estos indicadores propicia el establecimiento de un diagnóstico que sintetiza, en categorías, la degradación del territorio. De cierta manera, el diagnóstico da la medida de la eficiencia geoecológica del territorio, porque al ser tratados en términos de degradación, respondiendo a los indicadores analizados, resulta obvio que las unidades tratadas como muy degrada- das son las que resultan ineficaces desde el punto de vista geoecológico; es decir, paisajes cuyos componentes han perdido la posibilidad de autorrenovación y autorregeneración, toda vez que el hombre truncó su tendencia evolutiva.

De acuerdo con todo lo anterior, se estimó la estabilidad natural de los diferentes unidades de paisajes, a partir de la cuantificación de la Estabilidad Interna, la Estabilidad Externa y la Estabilidad Natural. Con ello se elaboró un mapa de Diagnóstico Geoecológico que expresa el grado de degradación de las diferentes unidades del parque. 
Parque Metropolitano de La Habana - Diagnóstico Geoecológico

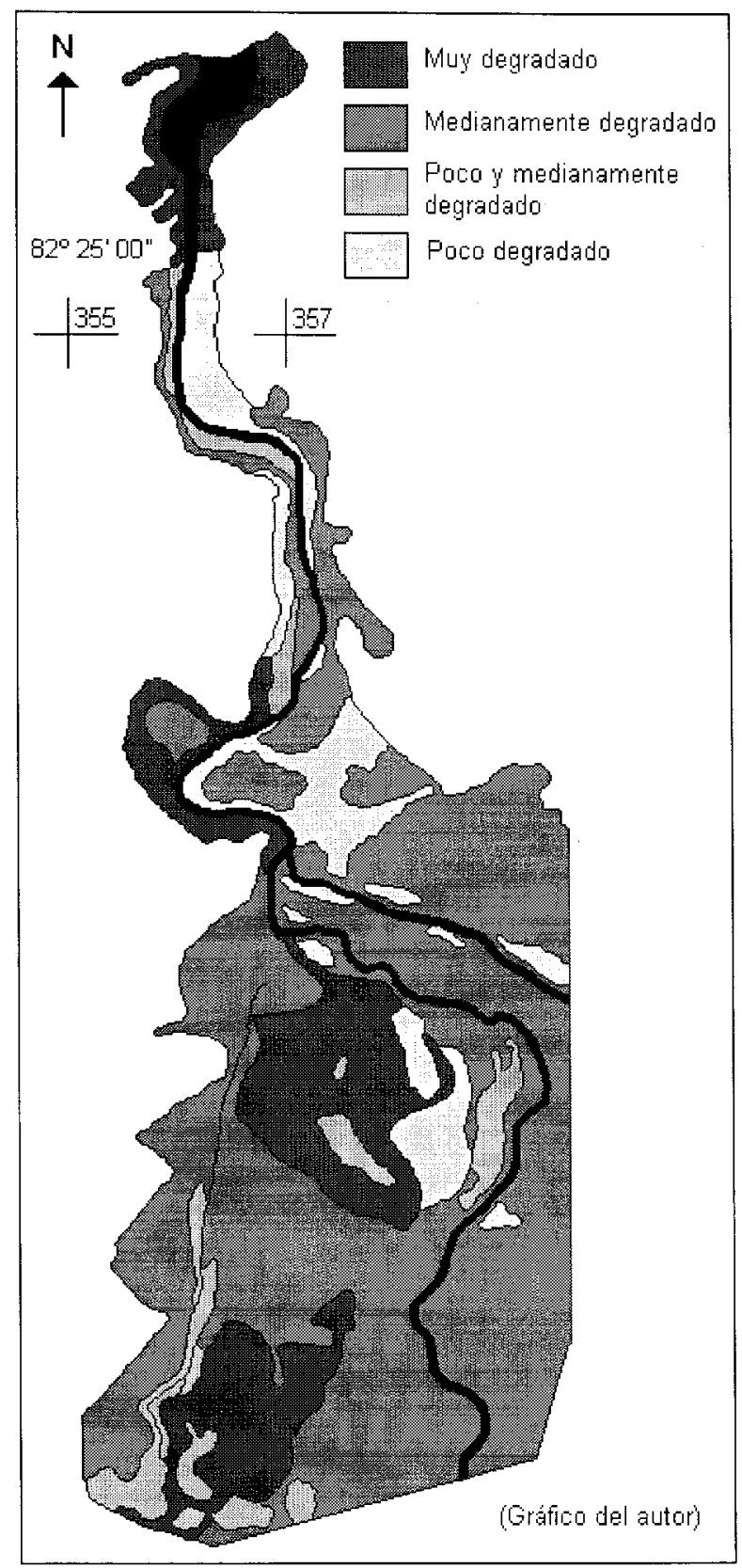

